\title{
Estrategias corporativas y reconfiguración de las cadenas de proveeduría del vestido hacia Estados Unidos: el caso de la firma Levi Strauss \& Co.
}

\author{
Guadalupe García de León P.*
}

Resumen. Este artículo presenta un recuento de las estrategias corporativas de la firma Levi Strauss \& Co., empresa que ha tenido un papel protagónico en cada una de las fases del proceso de integración de México a la producción global de prendas de vestir: la integración bajo los programas de producción compartida; la primera etapa del TLCAN; y la etapa actual de caída de las exportaciones mexicanas y reposicionamiento de China. El argumento central es que analizando las estrategias competitivas de las firmas líderes de las redes globales del vestuario -como es el caso de Levi's-, nos podemos acercar a la lógica de reestructuración de estas redes y entender los términos cambiantes de inserción internacional de los países y firmas involucradas, como es el caso de México.

Palabras clave: Levi Strauss \& Co., redes globales del vestido, TLCAN, comercio internacional, estrategias competitivas.

Abstract. This article presents a summary of the corporate strategies of the Levi Strauss \& Co. firm, a company that has had an important role in each one of the phases of Mexico's process of integration into the global apparel production: the integration under the shared-production programs, the first stage of NAFTA, and the current stage of decline of Mexican exports and re-positioning of China. The central argument is that, by analyzing the competitive strategies of the leading firms of global apparel networks - as in the case of Levi's -, we can get closer to the restructuring logic of these networks and understand the changing terms of the international insertion of the countries and firms involved, as in the case of Mexico.

Keywords: Levi Strauss \& Co., global apparel networks, NAFTA, international trade, competitive strategies.

Artículo recibido el 19 de noviembre de 2008 Artículo aceptado el 19 de junio de 2009

\footnotetext{
* Doctora en Relaciones Internacionales Transpacíficas. Departamento de Economía de la Universidad de Sonora. Correo electrónico: ggarcia@guaymas.uson.mx y guadalupe.garciadeleon@gmail.com
} 


\section{Introducción}

La internacionalización de la industria mexicana del vestido es un proceso que arranca desde la década de los sesenta del siglo pasado, al amparo del llamado "régimen de producción compartida", ${ }^{1}$ el cual generó las condiciones para el surgimiento de las primeras maquilas de la confección en el norte del país. Sin embargo, es hasta la segunda mitad de los años ochenta cuando cobra fuerza el desarrollo de una industria exportadora de prendas de vestir vinculada al mercado estadounidense, fenómeno que combina los procesos de crisis y apertura externa por el lado mexicano, y las necesidades de reestructuración de la industria textil y del vestido en Estados Unidos frente a la creciente ola de importaciones baratas procedentes de Asia.

Así, desde mediados de la década de los ochenta algunas de las mayores firmas estadounidense manufactureras de prendas de vestir empezaron a recurrir en forma creciente al ensamblaje offshore en México, Centroamérica y el Caribe, tomando ventaja de los bajos salarios, de la proximidad geográfica, y del conjunto de incentivos establecidos por los gobiernos de estos países para atraer inversiones extranjeras a sus zonas de procesamiento para la exportación. Una de las principales actividades que se mueven hacia esta región es la manufactura de prendas de algodón, especialmente pantalones, mediante las operaciones de los mayores fabricantes de este tipo de prendas, como es el caso de Levi Strauss \& Co. y VF Corporation. La operación del Tratado de Libre Comercio de América del Norte (TLCAN) abrió una nueva etapa en la historia del despliegue territorial de la producción de prendas de vestir dentro del continente americano, convirtiéndose en un importante catalizador del proceso de integración de México a los circuitos internacionales de esta industria, y modificando las formas y los mecanismos puestos en juego para dicha integración.

\footnotetext{
${ }^{1}$ Dentro de la industria del vestido, la provisión 807 , también denominada "producción compartida" y clasificada bajo el título 9802 en el actual Sistema Armonizado de Tarifas de Estados Unidos (HTSUS, por sus siglas en inglés), permite que el material que ha sido cortado en Estados Unidos sea exportado provisionalmente a otros países para su ensamblaje, y reimportado de nuevo pagando aranceles sólo por el valor agregado en las actividades de costura.
} 
Ubicándonos en este marco de referencia, el presente artículo analiza las estrategias corporativas de la firma Levi Strauss \& Co. (LS\&CO), empresa que ha tenido un papel protagónico en cada una de las fases de la trayectoria de integración de México a las redes globales de la industria del vestido: la integración bajo los programas de producción compartida; la primera etapa del TLCAN; y la etapa actual de caída de las exportaciones mexicanas y reposicionamiento de China. El argumento central es que analizando las estrategias competitivas de las firmas líderes de las redes globales del vestuario -como es el caso de Levi's-, nos podemos acercar a la lógica de reestructuración de estas redes y entender los términos cambiantes de inserción internacional de los países y firmas involucradas, como es el caso de México.

El texto está organizado en cuatro apartados. El primero proporciona los elementos que dan sustento al enfoque analítico de este estudio, basado en el concepto cadenas globales de valor. Utilizando este enfoque, la segunda sección ubica la firma Levi Strauss en el marco de la cadena de valor del vestuario orientada al mercado estadounidense. La tercera sección aborda el despliegue de las estrategias corporativas de esta firma a lo largo de las dos últimas décadas, identificando el tipo de redes de suministro que se desarrollan en México así como los términos de inserción de este país que dichas estrategias promueven. Se concluye con el apartado de conclusiones.

\section{El papel protagónico de los agentes líderes de las cadenas globales de valor}

El soporte conceptual de este estudio se apoya en el enfoque cadenas globales de valor (García de León, 2008), el cual constituye un esfuerzo teórico desarrollado con el propósito de proveer elementos de comprensión sobre la estructura y el funcionamiento de las redes de producción y distribución en la nueva arquitectura del capitalismo global. Desde este enfoque, dichas redes constituyen las estructuras organizativas fundamentales a través de las cuales las economías nacionales se vinculan e interactúan con el sistema internacional. Los vínculos son múltiples y se establecen en los distintos niveles de operación: local, regional, global. Las configuraciones políticas, económicas y so- 
ciales de la sociedad global son el resultado de la interacción entre estos distintos niveles de actividad, cuyas conexiones e interdependencias construyen la compleja trama de las diferentes manifestaciones del capitalismo local e internacional.

La estructura analítica de este enfoque se construye sobre el concepto básico cadena de valor, el cual se sustenta en la idea de que los bienes y servicios consumidos en los mercados finales son producto de varias etapas discretas y divisibles de actividad económica:

La cadena de valor describe el ámbito total de actividades requeridas para llevar un producto o servicio desde su concepción, a través de las fases intermedias de producción (la cual incluye una combinación de transformaciones físicas y el abastecimiento de varios servicios al productor), distribución al consumidor final, y su disposición final después de su uso (Kaplinsky, 2000:8).

Una fuente primaria de la reciente preponderancia de las cadenas de valor como herramienta analítica deriva del trabajo de Michael Porter, quien en el transcurso de la década de los ochenta introdujo este concepto para examinar las fuentes de la ventaja competitiva de una empresa y las estructuras fundamentales que son requeridas para el escalamiento de las capacidades nacionales. Al trabajo desarrollado por Porter sobre las cadenas de valor, se le reconocen dos aportaciones de gran riqueza analítica: el enfatizar la estructura secuencial e interconectada de las actividades de agregación de valor, con cada vínculo o elemento en la cadena agregando valor al proceso (Henderson, 2002:4); y la distinción que establece entre las actividades primarias (tangibles), directamente involucradas en el flujo material de un producto (su creación física, su venta y transferencia al comprador, así como la asistencia posterior a la venta), y las actividades de apoyo a esas actividades primarias y a la cadena en su conjunto (desarrollo tecnológico, desarrollo de marcas, administración de recursos humanos, administración general, planeación, finanzas, contabilidad, administración de calidad, etcétera) (Porter, 2000).

Porter llama la atención sobre el hecho de que en muchas ocasiones el mayor valor agregado proviene de este último tipo de actividades 
(servicios de apoyo o actividades intangibles), así como de la optimización y coordinación de las relaciones o vínculos establecidos entre las actividades de una cadena. Las etapas donde se realiza propiamente la producción son, pues, consideradas sólo una parte del conjunto de eslabones de agregación de valor, lo cual permite evitar algunas deficiencias que resultan de un enfoque analítico estrecho interesado exclusivamente en la manufactura.

Los elementos desarrollados por Porter para el análisis de las cadenas de valor al nivel de la firma han sido retomados dentro del enfoque de las cadenas globales de valor para el estudio de las redes globales de producción y comercialización de productos específicos, con el propósito de observar los vínculos entre actividades económicas, su difusión espacial, y los roles y relaciones de poder que existen entre varios actores de una industria.

Gereffi utiliza el término global commodity chains, y define a estas cadenas como "el conjunto de actividades involucradas en el diseño, producción y comercialización de un producto" (1999:3). A diferencia de la cadena de valor de Porter, el concepto global commodity chain incorpora explícitamente la dimensión internacional dentro de su análi$\mathrm{sis}^{2}{ }^{2}$ reflejando la atención primaria de esta propuesta analítica sobre la emergencia de sistemas de manufactura que están dispersos e integrados a una escala mundial. Desde la perspectiva de la cadena de valor, es posible fragmentar partes de la cadena para analizar más de cerca la forma en que se organizan las actividades internacionalmente. Cada nodo, señala Gereffi (1997:43), es en sí mismo una red, conectada a otros nodos involucrados en actividades relacionadas. Las cadenas de valor son globales y se estructuran de manera compleja, a través de un gran número de redes.

\footnotetext{
${ }^{2}$ Según apreciación del propio Gereffi, el enfoque global commodiy chains difiere al menos en cuatro aspectos respecto al concepto relacionado cadenas de valor de Porter: 1. incorpora una dimensión internacional explícita dentro del análisis; 2. se enfoca en el poder ejercido por las firmas líderes en diferentes segmentos de la cadena del producto, e ilustra cómo el poder se transforma a lo largo del tiempo; 3 . considera la coordinación de la cadena en su totalidad como una fuente crucial de ventaja competitiva que requiere utilizar las redes como un valor estratégico; y 4. considera el aprendizaje organizacional como un mecanismo crítico por medio del cual las firmas tratan de mejorar o consolidar sus posiciones dentro de la cadena (Gereffi, 1999:3).
} 
Dentro del enfoque de cadenas globales de valor, la llamada gobernanza constituye uno de los aspectos a los que se ha conferido mayor importancia en el análisis de las formas de organización de las firmas e industrias globalizadas. Para Ernst (1997:17) el término governance hace referencia a cómo "dentro de una red particular, el control y la coordinación es ejercido y por quién". Por su parte, Appelbaum y Gereffi (1994:43) definen las estructuras de gobierno como "las relaciones de autoridad y poder que determinan cómo los recursos financieros, materiales y humanos son localizados y fluyen dentro de una cadena".

Respecto a los agentes que influencian o definen la distribución del control y las relaciones de poder a partir de las cadenas globales, mucha de la literatura ha centrado la atención en el papel crucial de las principales empresas multinacionales como fuerza conductora de la globalización al forjar vínculos a través de las firmas y fronteras nacionales.

Los agentes líderes de las cadenas globales ejercen la función de gobierno a partir de sus estrategias corporativas, las cuales tienen como principal objetivo el posicionamiento de la cadena en el mercado. Así, la firma líder tiene un papel decisivo en determinar qué se ofrece a los consumidores, esto es, qué productos están disponibles, cuándo, y sus características (calidad, apariencia, empaquetado, etcétera). Lo anterior incluye la interpretación de las tendencias del mercado y la especificación de cuáles productos deben elaborarse para ajustarse a dichas tendencias. Puede también incluir las especificaciones de cuáles procesos deben seguirse para elaborar dichos productos, o sólo dictar el qué y dejar el cómo bajo la responsabilidad del proveedor (Humphrey y Schmitz, 2000:3-4). La función de gobierno también involucra el establecimiento de sistemas de supervisión y control a lo largo de la cadena, especificando los sistemas de calidad de los proveedores y los procedimientos para monitorear su desempeño (Kaplinsky, 2000:13).

En ese sentido, las estrategias corporativas de las firmas líderes desempeñan un papel central en la forma en que la producción internacional es organizada. Estas decisiones influencian la estructura total de la cadena, el tipo y número de firmas involucradas, así como la distribución de funciones entre las mismas. También influencian deci- 
siones específicas sobre cuáles productores o exportadores son incluidos o excluidos de la cadena.

Los liderazgos en las redes globales se establecen por aquellos agentes que logran dominar los espacios de mayor valor agregado y rentas dentro de una cadena (Kaplinsky, 2000). Un aspecto importante derivado de lo anterior es el reconocimiento de que los agentes líderes pueden estar localizados en diferentes puntos de la cadena, no sólo en las actividades de producción:

Estas firmas líderes no son necesariamente las tradicionales manufactureras verticalmente integradas, ni necesitan aún estar involucradas en la fabricación de productos finales. Estas firmas pueden estar localizadas hacia arriba o debajo de la manufactura (como los diseñadores de moda o minoristas de etiquetas privadas en el vestuario), o pueden estar involucradas en el suministro de componentes críticos (como compañías de microprocesadores como Intel, o firmas de software como Microsoft dentro de la industria de computadoras) [...] Lo que distingue a las firmas líderes de sus seguidoras o subordinadas es que éstas controlan el acceso a los principales recursos (como diseño de producto, nuevas tecnologías, nombres de marca o demanda del consumidor) que generan los ingresos más rentables en la industria (Gereffi, 1999:3).

Dentro de las cadenas globales de suministro del vestido hacia Estados Unidos han surgido diferentes tipos de firmas líderes. En los segmentos dedicados a la comercialización y venta de estos productos se encuentran las gigantes de venta al menudeo o retailers (por ejemplo, Wal-Mart, K-Mart, J.C. Penney, Sears, etcétera), y las grandes compañías diseñadoras-comercializadoras de marcas (por ejemplo, Liz Claiborne, Polo Ralph Lauren, Nike, etcétera). Dentro de los segmentos involucrados en las fases de producción, destacan las grandes compañías manufactureras de prendas de vestir de marca (por ejemplo, Levi Strauss, Sara Lee, vF Corporation). Estas corporaciones minoristas, comercializadoras y fabricantes de marcas han desempeñado papeles de pivote en el establecimiento de redes de producción 
en una variedad de países exportadores, típicamente localizados en el tercer mundo (Gereffi, 1999).

\section{El posicionamiento de Levi Strauss \& Co. como agente líder dentro de las redes globales de suministro del vestido hacia Estados Unidos}

Como se señaló anteriormente, una consideración central en el concepto cadenas de valor es que los bienes y servicios consumidos en los mercados finales son producto de una estructura secuencial de agregación de valor. Desde esta perspectiva, considerada en su forma más general, la cadena de valor del vestuario abarca cuatro segmentos principales:

1. Producción de materias primas, como fibras naturales (algodón, lana, seda, etcétera) y productos químicos para la elaboración de fibras sintéticas (petroquímicos).

2. Producción de insumos y componentes, principalmente hilos y telas (de fibras naturales y sintéticas) elaborados por fábricas textiles. $^{3}$

3. Manufactura de prendas de vestir, que incluye todos los procesos de transformación de la tela y otros componentes, en prendas terminadas listas para su venta.

4. Comercialización, que incluye los canales de distribución y venta de los productos terminados (mayoreo y menudeo).

Cada uno de estos segmentos representa nodos de actividad de la cadena en su conjunto o, utilizando la terminología de Porter (2000), definen una función amplia o categoría genérica dentro de la secuencia de agregación de valor. Sin embargo, el proceso mediante el cual aun la más sencilla prenda de vestir pasa por las etapas anterior-

${ }^{3}$ Las prendas de vestir son producidas utilizando principalmente materiales textiles, los cuales incluyen telas tejidas, de tejido de punto y no tejidas, confeccionadas de algodón, otras fibras vegetales (por ejemplo, lino), lana, fibras sintéticas y seda. Los materiales no textiles utilizados en la manufactura del vestuario incluyen piel, hule y plásticos (US International Trade Commission, 1999). 
mente descritas se ha vuelto sumamente complejo en el nuevo orden internacional. El número de actividades y vínculos involucrados en la operación de cada uno de estos nodos puede ser potencialmente muy alto.

Dentro de las cadenas globales de valor del vestido, las redes de proveeduría o suministro se han concentrado fundamentalmente (aunque no exclusivamente) en la etapa de manufactura de estos productos (la cual incluye la gradación, trazado, corte, costura, terminados y empaquetado). En particular, la cadena de suministro de prendas de vestir hacia el mercado de Estados Unidos nos ofrece un panorama amplio, complejo y heterogéneo de redes de producción integradas por un gran número de compañías confeccionistas, sus contratistas y filas de subcontratación, ubicadas en una variedad de países alrededor del mundo. Dentro de esta red global de producción ${ }^{4}$ se realizan -en una forma crecientemente descentralizada y territorialmente dispersalas distintas actividades requeridas para la manufactura total de este tipo de bienes. Además, la fase de manufactura de prendas de vestir se vincula hacia atrás con las redes de proveedores de insumos y componentes, y hacia delante de la cadena, con las redes de distribución, comercialización y venta al menudeo (figura 1).

En Estados Unidos la industria de manufactura de prendas de vestir (rubro 315 del Sistema Norteamericano de Clasificación Industrial NAICS) se conforma por alrededor de 13 mil establecimientos que emplean a más de 300 mil trabajadores. La inmensa mayoría de estas firmas $(83 \%)$ y trabajadores (77\%) corresponden a la subdivisión 3152 de manufactura de corte y costura (US Census Bureau, 2002).

El proceso de manufactura dentro de esta industria se inicia con el diseño de la prenda que se va a confeccionar. A partir de ese diseño se elaboran patrones, los cuales son utilizados para cortar la tela. Para producir la prenda terminada, estas piezas de tela son normalmente ensambladas (costura), etiquetadas y empaquetadas. El segmento de ensamblado es el más intensivo y fragmentado en cuanto al uso de la mano de obra.

${ }^{4}$ Sobre estas bases, las redes globales de producción harían referencia a la fracción de las cadenas globales de valor involucrada con las etapas de manufactura de un producto. 
Figura 1. Cadena de valor del vestido

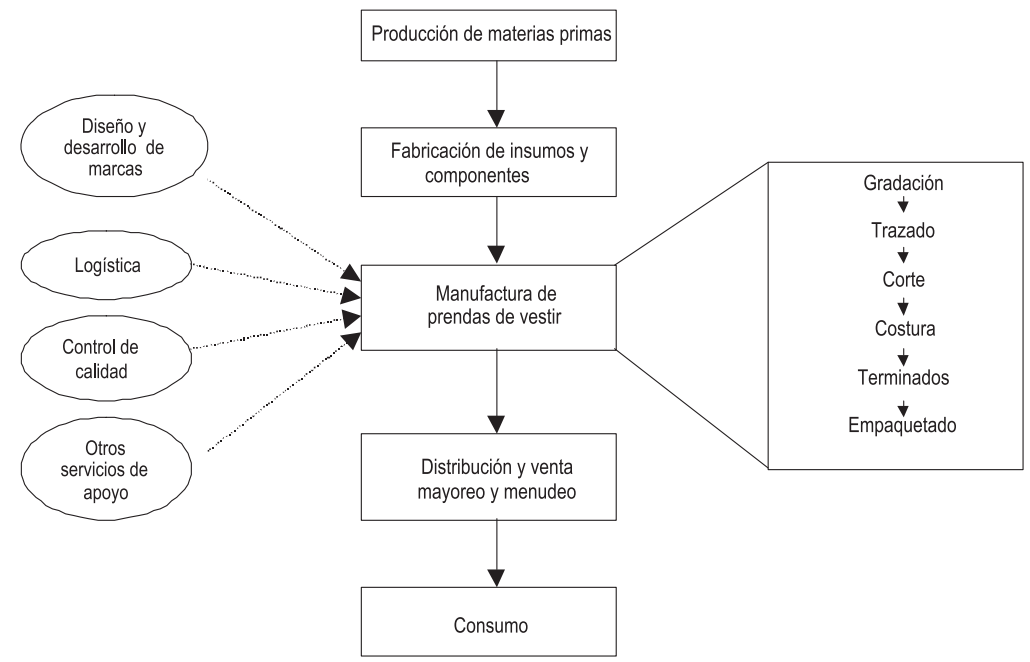

Fuente: Elaborado con base en el diagrama de Timothy Sturgeon (2001:4).

En su organización tradicional, las firmas manufactureras en Estados Unidos eran responsables de todas las fases de fabricación de la prenda. Sin embargo, actualmente el grado de involucramiento de estas firmas en las actividades de manufactura es variable. De hecho, como resultado de los constantes ajustes y reestructuraciones del sector, actualmente la mayoría de estas industrias está organizada bajo la modalidad de jobbers y contratistas. Los jobbers -también conocidos como outsourcing manufacturers- son firmas que diseñan y comercializan prendas de vestir, pero subcontratan su producción a terceros (los contratistas). La subcontratación puede incluir sólo algunas etapas del procesamiento de las prenda (normalmente las más intensivas en trabajo), o bien la contratación del servicio de "paquete completo", dentro del país o en el extranjero (US International Trade Commission, 1999).

La firma Levi Strauss \& Co. surge y se consolida como una de las mayores firmas manufactureras del vestido en Estados Unidos, sus- 
tentada en el florecimiento del mercado del jean (pantalón tipo vaquero fabricado de mezclilla), el cual constituye una de las categorías más fuertes de prendas de vestir dentro de ese mercado. De hecho, los jeans son la prenda de vestir más ampliamente consumida en Estados Unidos: se estima que cada año son comprados alrededor de 450 millones de pares de jeans. De acuerdo con datos de Cotton Incorporated (el órgano comercializador de la industria algodonera de Estados Unidos), el estadounidense promedio posee siete pares de jeans y compra dos por año. En Europa occidental este número es poco más de 4 y 1 , debido al mayor rango de ropa de vestir (Newbery, 2004).

La historia de la compañía Levi Strauss está ligada a la propia historia del surgimiento y consolidación del jean como un ícono de la sociedad norteamericana de la posguerra. La empresa fue fundada por el empresario judío del mismo nombre, nacido en Alemania y emigrado a Estados Unidos. Habiéndose establecido en San Francisco, Levi Strauss registra en 1873 -junto con el sastre italiano Jacob Davis- la patente del proceso y la marca del primer pantalón ribeteado de Levi's.

Levi's comenzó a vender sus jeans nacionalmente durante la década de los cincuenta. Para los años sesenta la mezclilla se había convertido dentro del mundo industrializado en un símbolo del estilo joven, activo e informal del American way of life. Había dejado de ser una tela de ropa de trabajo, para ser el ingrediente más importante y de moda en la confección de ropa casual de todos los tipos. A principios de los años setenta, la mezclilla azul índigo era considerada como "un fenómeno sin paralelo" para la juventud estadounidense, la tela top fashion mundial para pantalones (Downey, 2007).

Como resultado de una exitosa trayectoria de expansión global, para 1997 Levi Strauss \& Co. se encontraba posicionada como la mayor firma manufacturera de prendas de vestir en el mundo. Sus ventas anuales alcanzaban los 7 billones de dólares y 71\% de éstos correspondía a jeans o artículos relacionados. Sus gastos anuales de publicidad alcanzaban 300 millones de dólares en Estados Unidos y 200 millones en el exterior. Entre 1984 y 1997, el valor de mercado de la firma creció 105 veces, casi tanto como el gigante Microsoft. Sin embargo, en la última década sus ventas han caído de manera persisten- 
te, y Levi's ha dejado de ser la marca de jeans más vendida en Estados Unidos, al parecer, perdiendo la batalla y su antiguo liderazgo ante nuevos competidores y frente a un mercado de consumo cada vez más segmentado, exigente y dinámico.

\section{Estrategias corporativas de Levi Strauss y evolución de los vínculos con sus proveedores en México}

Primera etapa (1987-1997): el imperativo de reducir costos de producción

Históricamente, la estructura de abastecimiento de jeans provino directamente de las raíces "prácticas" de la ropa de trabajo americana. El producto era barato, y también eran baratas sus materias primas. La proximidad fue importante: las fábricas de mezclilla y de jeans se agruparon en cercanía a las regiones de cultivo de algodón. Hubo también una razón laboral, pues la mano de obra masculina prevaleció en las fábricas textiles de la mezclilla, y la mano de obra femenina se ocupó en las fábricas de jeans como operadora de máquinas de coser (Newbery, 2004). Este patrón territorial de organización de la cadena de suministro del jean en Estados Unidos ha ido cambiando de manera progresiva en respuesta a las también cambiantes condiciones de la competencia. Esto fue especialmente evidente a partir de la segunda mitad de los años ochenta, cuando la creciente ola de importaciones asiáticas de bajo costo puso en jaque a la industria textil y del vestuario de Estados Unidos.

Desde la década de los setenta Estados Unidos comenzó a registrar un crecimiento constante de sus importaciones de prendas de vestir. Éstas se expandieron especialmente durante los años ochenta, pasando de 5518 millones de dólares en 1980 a casi 22 mil millones en 1990, llegando a representar para finales de esa década una tercera parte del consumo total de ropa en Estados Unidos (Bonacich y Waller, 1994). Es importante señalar que el aumento de las importaciones fue inducido por las mismas firmas minoristas y comercializadoras de marcas estadounidenses, las cuales comenzaron a descansar cada vez en mayor medida en el abastecimiento externo mediante la compra de bienes elaborados por los productores del este asiático. 
La competencia de precios de las prendas importadas colocó a los productores locales -textiles y manufactureros- bajo una tremenda presión por reducir sus costos de operación. Se abre así una etapa crítica para este sector en Estados Unidos, caracterizada por un gran número de quiebras y caída drástica del empleo, situación que condujo a un conjunto de respuestas estratégicas por parte de las empresas y el propio gobierno de ese país, las cuales estaban orientadas tanto a contener las importaciones asiáticas como a fortalecer la competitividad internacional de su industria doméstica.

En particular, las estrategias competitivas seguidas por Levi Strauss \& Co. durante esta etapa abarcaron dos frentes: $a$ ) la racionalización de los procesos de producción en sus fábricas, a partir de la introducción de innovaciones tecnológicas y organizacionales; y $b$ ) el translado de las etapas intensivas en trabajo (costura) hacia países de bajos salarios, principalmente México y la región del Caribe.

A) Estrategias de racionalización de la producción: los programas AMS y FAST

La necesidad de desarrollar ventajas competitivas distintas a las salariales (en las cuales los productores domésticos tienen desventajas respecto a los países de bajos salarios) ha definido como enfoque estratégico central de la industria del vestido en Estados Unidos la capacidad de dar una "respuesta rápida" a las señales de la demanda. Las estrategias QR (Quick Response) se basan en el reconocimiento de la creciente complejidad y diversificación de este mercado, y en la ventaja que otorga la proximidad con los propios consumidores estadounidenses. Esta cercanía permite a las firmas domésticas monitorear los cambios en la demanda y responder a los mismos en forma rápida, a partir de formas de organización flexibles y especializadas.

Las estrategias de racionalización de la producción impulsadas por Levi Strauss a través de los programas AMS y FAST se orientaban en esta dirección. El Alternative Manufacturing Systems (AMS) ${ }^{5}$ fue un

${ }^{5}$ El AMS fue implementado por Levi's a principios de los años noventa en la mayoría de las plantas de costura de Estados Unidos, así como en sus instalaciones de Canadá y Brasil (Levi Strauss \& Co., Informe 10-K, 1993 ). 
enfoque de manufactura modular basado en equipos de trabajo, que sustituyó la línea tradicional de ensamblado. El trabajo de producción en la industria del vestido se ha desarrollado tradicionalmente bajo el sistema de líneas de ensamblado, en el cual el trabajador realiza una misma operación de ensamblaje cuando las piezas se mueven a lo largo de la línea de producción. El sistema modular, por contraste, incorpora la filosofía organizativa de los equipos de trabajo, y los operadores son entrenados en casi todas las funciones requeridas para ensamblar una prenda de vestir. Como resultado, el sistema modular es más flexible, productivo y sensible a los cambios en la demanda (US Department of Labor, 2000). Adicionalmente, Levi's implementó en Estados Unidos el programa denominado FAST, para vincular plantas específicas de costura a ciertos centros de terminados y centros de servicio al cliente, con el objetivo de reducir sus tiempos de gestión y procesamiento y lograr una mayor velocidad de respuesta para atender los pedidos de sus clientes (Levi Strauss \& Co., Form $10 \mathrm{~K}$ Annual Report, 1993). ${ }^{6}$

\section{B) Relocalización hacia México, Centroamérica y el Caribe}

Otro importante frente de reestructuración de la industria norteamericana del vestido desde la segunda mitad de la década de los ochenta lo constituye el reforzamiento de la estrategia de ensamblaje en el extranjero, mediante el traslado de las etapas intensivas en trabajo (costura) hacia países donde el costo laboral es menor que en Estados Unidos, utilizando para ello la provisión del Ítem 807, conocido como "producción compartida".

Levi's fue una de las grandes firmas estadounidenses manufactureras de prendas de vestir que utilizó la estrategia de relocalización hacia México y la Cuenca del Caribe. En esos años, esta empresa estaba posicionada como la firma de ropa más importante en Estados Unidos, y el mercado de jeans seguía en ascenso, con un crecimiento de $10 \%$ anual. Sin embargo, esto no era suficiente ante una oferta tam-

${ }^{6}$ Los informes anuales de Levi Strauss \& Co. referidos en este estudio corresponden a los reportes 10-K sobre operaciones anuales, presentados ante la Us Securities and Exchange Commission (SEC). 
bién creciente de productos a menores precios. La ropa casual requiere de mayores tiempos de costura y terminado, por consiguiente, es difícil mantener un nivel competitivo de costos cuando se producen dentro de las instalaciones de la firma en Estados Unidos (Levi Strauss \& Co., Informe 10-K, 1993). Había la necesidad de reducir costos para mantener su filo competitivo en un mercado en constante expansión, que amenazaba con ser arrebatado por los nuevos competidores de dentro y fuera del país.

En estas circunstancias, Levi's comenzó a utilizar cada vez en mayor medida a contratistas independientes para las funciones de costura y terminados. Para 1994, aproximadamente 50\% de las unidades producidas por Levi's dentro de Estados Unidos fue manufacturado por contratistas independientes; mientras que esa proporción se ubicaba en $45 \%$ de su producción en el extranjero (Levi Strauss \& Co., Informe 10-K, 1995).

Para 1990, la firma recurría al servicio de maquila de sus marcas Levi's, Docker y Britania, tanto en México como en Guatemala, Costa Rica y República Dominicana, a través de contratistas independientes a los cuales proveía los materiales e insumos de producción. Para dar una idea de las ventajas de producir en estos países, vale hacer referencia al enorme diferencial del salario por hora para los operadores de costura. En 1991, dicho salario era de 6.77 dólares en Estados Unidos, contra 1.09 dólares en Costa Rica, 0.88 en México, 0.75 en República Dominicana, y tan sólo 0.45 en Guatemala (Bonacich y Waller, 1994:32).

Segunda etapa: las estrategias a partir de 1997

A pesar de las dificultades para mantenerse competitivo en un entorno de crecientes presiones, las ventas de Levi's registraron un continuo ascenso hasta 1996, año en que alcanzaron la cifra récord de 7136 millones de dólares en ventas netas, proceso ligado a la constante expansión del mercado y crecimiento de los productos de la marca.

Sin embargo, a partir de 1997 las ventas comenzaron a descender, tendencia que se acentúa en los siguientes años. En 2001 las ventas totales de la firma habían caído $40 \%$ respecto al nivel alcanzado cinco 
años atrás, y desde entonces no han podido repuntar de manera significativa (cuadro 1).

La anterior situación revela las crecientes dificultades enfrentadas por Levi's para encarar los retos competitivos que implica la propia evolución del mercado del jean. Por una parte, este mercado ha vivido un fenómeno de polarización hacia los dos extremos de su espectro: hacia las marcas masivas de Wal-Mart, y hacia las marcas de grandes diseñadores, como Armani. El mercado medio masivo, el cual consume Levi's, Lee y Wrangler, ha sido golpeado por este fenómeno. Además, dentro de su tradicional segmento de mercado, las marcas de Levi's enfrentan una fuerte competencia por parte de grandes diseñadores-comercializadores que han venido ganando terreno, como

Cuadro 1. Evolución de las ventas netas totales de Levi Strauss, 1990-2007

\begin{tabular}{lcc}
\hline Año & $\begin{array}{c}\text { Ventas netas } \\
\text { millones de dólares }\end{array}$ & $\begin{array}{c}\text { Índice de ventas netas } \\
1996=100\end{array}$ \\
\hline 1990 & 4247 & $60 \%$ \\
1991 & 4903 & $69 \%$ \\
1992 & 5570 & $78 \%$ \\
1993 & 5893 & $83 \%$ \\
1994 & 6074 & $85 \%$ \\
1995 & 6708 & $94 \%$ \\
1996 & 7136 & $100 \%$ \\
1997 & 6862 & $96 \%$ \\
1998 & 5959 & $84 \%$ \\
1999 & 5139 & $72 \%$ \\
2000 & 4645 & $65 \%$ \\
2001 & 4259 & $60 \%$ \\
2002 & 4146 & $58 \%$ \\
2003 & 4091 & $57 \%$ \\
2004 & 4073 & $57 \%$ \\
2005 & 4151 & $58 \%$ \\
2006 & 4107 & $58 \%$ \\
2007 & 4266 & $60 \%$ \\
\hline
\end{tabular}

Fuente: US Securities and Exchange Commission (SEC), Electronical Data Gathering Analysis and Retrieval (EDGAR) database. Levi Strauss \& Co., informes anuales 10-K, varios años, http://www.sec.gov/. 
es el caso de Gap que actualmente controla una porción significativa del mercado medio masivo.

La crisis originada por la caída de sus ventas orilló a Levi Strauss a ponerse en manos de consultores externos para su reestructuración. La orientación central de dicha reestructuración ha sido transformar de raíz su naturaleza como empresa manufacturera de prendas de vestir, desprendiéndose de sus fábricas y trabajadores para convertirse en una compañía centrada en marcas (brand-centered). Lo anterior ha abarcado un conjunto de estrategias corporativas entre las que destacan las siguientes:

A) Segmentación de marcas y diversificación de canales de comercialización

Con el propósito de lograr mejorar su posicionamiento en el mercado, Levi's ha puesto en el centro de sus estrategias competitivas la llamada "segmentación" de sus marcas, la cual ha sido considerada absolutamente crítica para responder a la propia segmentación del mercado. En particular, el objetivo ha sido la transición de la marca ícono "Levi Strauss" hacia el mercado masivo de jeans, a través de su segmento Levi Strauss Signature, el cual comenzó a venderse en las tiendas WalMart a partir de 2002.

Esta estrategia por parte de la firma obedece a que el mercado masivo del jeans es uno de los pocos canales que muestran crecimiento en venta de estos productos. Se estima que en Estados Unidos cada semana compran en las tiendas de descuento 160 millones de consumidores, y ése es el mercado objetivo para Levi's. En especial, la oferta dominante de la marca Signature es de jeans para hombres, aunque dicha marca abarca pantalones y tops para hombres, mujeres y niños.

Si bien ésta no es la primera ocasión en que Levi's explora el mercado de masas, pues durante los años ochenta fue realizado un movimiento similar con la etiqueta Britania, posteriormente vendida a VF Jeanswear; sin embargo, el lanzamiento de los jeans Signature es el primer intento de comercializar su marca líder directamente dentro del mercado de consumo masivo. Los resultados parecen respaldar lo certero de esta medida, no obstante las especulaciones que la misma 
despertó. A sólo dos años de su lanzamiento, la marca Signature alcanzó a representar $10 \%$ de las ventas netas totales de la corporación a nivel global, y casi 15\% de las ventas en la región norteamericana, la cual incluye Estados Unidos, México y Canadá, porcentaje que en términos absolutos significa alrededor de 350 millones de dólares anuales.

Muy ligado con la estrategia de segmentación de marcas, Levi Strauss ha seguido también la estrategia de apertura de nuevos canales de comercialización y medios de distribución. En este ámbito, la orientación central parece ser el tomar un lugar en el campo de ventas al menudeo, esto es, ir directo al consumidor. Dicho movimiento ha incluido la apertura de tiendas para la venta exclusiva de sus marcas Levi's y Dockers, así como el manejo de la figura de franquicias para ampliar su red mundial de distribución. Para 2006 Levi's tenía -además de las 138 tiendas de su propiedad-, más de 1100 tiendas bajo franquicia alrededor del mundo. Además, Levi's ha creado toda una red de tiendas de venta directa desde la fábrica, los llamados "factory outlets", los cuales constituyen un canal de distribución al menudeo en rápido crecimiento en Estados Unidos.

B) Desverticalización de operaciones y subcontratación en el extranjero

Levi Strauss fue uno de los últimos fabricantes de jeans en mantener un alto porcentaje de su producción domésticamente, conservando salarios de 9 a 14 dólares la hora (Colwell, 2002). No obstante, la competencia finalmente se impuso sobre esta política de mantener las plantas de producción y los empleos dentro del territorio estadounidense. Como parte de sus planes de reestructuración, entre 1998 y 2002 Levi's cerró 30 de las 32 plantas de producción y acabados de su propiedad en Estados Unidos, desplazando una fuerza laboral de casi 17 mil trabajadores, lo que representó una reducción del $75 \%$ respecto a su plantilla de 1997, la cual ascendía a 22600 trabajadores (Levi Strauss Co., informes anuales 10-K, varios años). Datos para 2007 (cuadro 2) muestran que en la actualidad la firma no posee ninguna planta de producción en Estados Unidos, en donde las instalaciones de su propiedad se han reducido a sólo cinco plantas de distribución, y se ha desprendido 
también de sus activos de producción en el extranjero, en donde sólo mantiene seis plantas de manufactura y almacenes.

La otra cara del proceso de desverticalización de Levi's ha sido el aumento de la subcontratación a terceros de la producción de prendas que antes realizaba en sus propias fábricas. Para 2004, del total de productos comercializados por Levi's, 92\% era manufacturado bajo contrato por productores independientes, y de este porcentaje, $62 \%$ correspondía a contratos de "paquete completo", es decir, al servicio de manufactura en el cual el proveedor asume la responsabilidad de organizar todas las actividades de producción hasta obtener el producto terminado, conforme a los requerimientos de costo, calidad y calendarios de entrega exigidos por su cliente. Asimismo, cada vez en mayor medida esta subcontratación se realiza en el extranjero, de tal manera que para 2004 los pedidos colocados dentro de Estados Unidos y Canadá representaron sólo $3.4 \%$ del total contratado por la firma.

Durante los primeros años de operación del TLCAN, México fue uno de los destinos principales de esta relocalización de la producción de

Cuadro 2. Instalaciones propiedad de Levi Strauss en Estados Unidos y el extranjero

\begin{tabular}{|c|c|c|c|c|c|c|c|c|}
\hline & \multicolumn{2}{|c|}{1995} & \multicolumn{2}{|c|}{2001} & \multicolumn{2}{|c|}{2004} & \multicolumn{2}{|c|}{2007} \\
\hline & Núm. & $\%$ & Núm. & $\%$ & Núm. & $\%$ & Núm. & 2. $\%$ \\
\hline \multicolumn{9}{|c|}{ Manufactura y almacenes } \\
\hline Estados Unidos & 44 & $46 \%$ & 8 & $18 \%$ & 0 & $0 \%$ & 0 & $0 \%$ \\
\hline Otros países & 22 & $23 \%$ & 14 & $32 \%$ & 5 & $26 \%$ & 6 & $33 \%$ \\
\hline Total & 66 & $69 \%$ & 22 & $50 \%$ & 5 & $26 \%$ & 6 & $33 \%$ \\
\hline \multicolumn{9}{|l|}{ Distribución } \\
\hline Estados Unidos & 8 & $8 \%$ & 4 & $9 \%$ & 4 & $21 \%$ & 5 & $28 \%$ \\
\hline Otros países & 21 & $22 \%$ & 18 & $41 \%$ & 10 & $53 \%$ & 7 & $39 \%$ \\
\hline Total & 29 & $31 \%$ & 22 & $50 \%$ & 14 & $74 \%$ & 12 & $67 \%$ \\
\hline Total de instalaciones & 95 & $100 \%$ & 44 & $100 \%$ & 19 & $100 \%$ & 181 & $100 \%$ \\
\hline
\end{tabular}

Fuente: US Securities and Exchange Commission (SEC), Electronical Data Gathering Analysis and Retrieval (EDGAR) database. Levi Strauss \& Co. Informes anuales 10-K, varios años, http://www.sec.gov/. 
Levi's hacia el exterior. Asimismo, bajo el nuevo marco libre de cuotas y aranceles que establece el TLCAN y conforme a las propias estrategias de reestructuración ya mencionadas, la firma modifica el tipo de órdenes de producción que coloca entre sus contratistas ubicados en México, saliendo del esquema maquilador para demandar servicios más integrados de manufactura como el "medio paquete"7 y el servicio de "paquete completo". De hecho, buena parte de las plantas cerradas y trabajadores desplazados en Estados Unidos como parte de los planes de desverticalización de esta empresa durante la década de los noventa, encuentran su contraparte en la proliferación -al otro lado de la frontera- de nuevas fábricas manufactureras y redes integradas de producción creadas con el propósito de atender las órdenes de trabajo colocadas por esta importante corporación.

Sin embargo, las ventajas del TLCAN parecen no haber sido suficientes para mantener el posicionamiento de México como principal región de proveeduría de esta firma. En los últimos años ha sido evidente el movimiento de las fuentes de suministro hacia la región asiática, especialmente a China. Datos de la empresa muestran que la región asiática incrementó de 27 a $40 \%$ su participación en el outsourcing total de Levi's, aumento que se corresponde con un descenso de la participación de la región latinoamericana, que en ese mismo lapso pasó de 59 a 40\% (Levi Strauss \& Co. Form 10-K Annual Reports).

Como podemos apreciar, el nuevo esquema de proveeduría configurado en respuesta a las propias necesidades estratégicas de la firma, ha desplazado el patrón maquilador en el cual México se especializó. El nuevo perfil de negocios de Levi's, que ha dejado de ser propiamente un fabricante para dedicarse a la administración de sus marcas, ha configurado un nuevo patrón de suministro en donde el origen geográfico de sus proveedores no es relevante; lo importante es contar con una base de contratistas altamente calificados que se ajusten a los requerimientos de costos, calidad, flexibilidad y veloci-

\footnotetext{
${ }^{7}$ En el servicio de "medio paquete", también conocido como cut-make-trim, la firma contratante retiene la propiedad de la tela a lo largo del proceso productivo como en el esquema maquilador, pero los servicios de transformación ofrecidos por el contratista incluyen también las etapas de corte y terminados; por ejemplo, la aplicación de cierres y el lavado posterior de la prenda en el caso de los jeans.
} 
dad de respuesta hacia el mercado, y con capacidad de brindar el servicio de "paquete completo", incluyendo en ocasiones hasta el propio diseño de las prendas. Y frente a este nuevo esquema, los proveedores asiáticos, y especialmente China, están ganando la batalla a México. Datos para abril de 2008 (cuadro 3) muestran que el número de fabricas que confeccionan prendas para Levi's en México es 29, frente a un número casi 10 veces mayor (269) de las localizadas en China.

La relación entre las estrategias competitivas emprendidas por la firma Levi Strauss y las nuevas pautas de suministro global que favorecen la región asiática sirven para ilustrar los problemas enfrentados por la industria textil y del vestido en México para seguir posicionada como principal proveedora de algunas de las categorías de prendas de vestir más fuertes en el mercado estadounidense, como es el caso de los jeans.

Hasta ahora, México continúa como el mayor exportador de pantalones y shorts de algodón (en donde se incluyen los jeans) a Estados

Cuadro 3. Fábricas proveedoras de Levi's en México y China. Datos a abril de 2008

\begin{tabular}{lclr}
\hline \multicolumn{1}{c}{ México } & & & China \\
& Número & Ubicación & Número \\
\hline Aguascalientes & 6 & Beijing & 2 \\
Chihuahua & 2 & Fujian & 16 \\
Coahuila & 1 & Guangdong & 114 \\
Distrito Federal & 2 & Hebei & 1 \\
Durango & 2 & Hubei & 2 \\
Estado de México & 2 & Jiangsu & 44 \\
Guanajuato & 3 & Liaoning & 5 \\
Hidalgo & 1 & Shandong & 39 \\
Puebla & 6 & Shanghai & 13 \\
Querétaro & 3 & Suzhou & 1 \\
Yucatán & 1 & Tianjin & 2 \\
& & Xinjiang & 1 \\
Total México & 29 & Zhejiang & 29 \\
\hline
\end{tabular}

Fuente: Levi Strauss \& Co., Supplier List, abril de 2008, http:// www.levistrauss.com/Downloads/FactoryList.pdf (3 de octubre de 2008). 
Unidos en la categoría para hombres, aunque China ha venido de manera consistente aumentando su participación y desplazando a otros importantes competidores como República Dominicana. Sin embargo, las exportaciones de pantalones y shorts de algodón para mujer por parte de China sobrepasaron a las de México entre 2004 y 2005, alcanzando para 2007 una participación de $16 \%$ en ese mercado (cuadro 4). Hasta el momento, estos productos continúan bajo cuotas para China, lo cual hace pensar en el predominio definitivo por parte de este país una vez que éstas expiren.

\section{Conclusiones}

Las estrategias corporativas de la firma Levi Strauss \& Co. para hacer frente a un mercado cada vez más competido, segmentado y volátil, constituyen la principal fuerza reconfiguradora de las estructuras internacionales a partir de las cuales se realiza la producción y distribución de las prendas de vestir fabricadas bajo sus marcas.

En los últimos 20 años, la inserción de México como abastecedor de esta firma ha vivido dos grandes etapas. La primera, bajo el esquema maquilador de suministro, respondió a una estrategia de reducción de costos para hacer viable una base manufacturera textil y de la confección que en Estados Unidos estaba siendo amenazada por la ola creciente de importaciones de dichos productos desde la región asiática.

La segunda etapa, bajo el TLCAN, se corresponde con un nuevo esquema de suministro bajo la modalidad de "paquete completo". Aunque en sus inicios esta nueva fase fue vista como una oportunidad para escalar las propias redes instaladas en México, actualmente resulta evidente que se está realizando un creciente desplazamiento de los proveedores localizados en este país hacia fuentes de la región asiática, principalmente de China.

El modelo de integración de México a las redes de suministro de prendas de vestir bajo el TLCAN no responde actualmente a las necesidades estratégicas de las grandes firmas manufactureras de prendas de vestir como es el caso de Levi's. Por una parte, México no representa ya una ubicación importante para las necesidades de suministro 


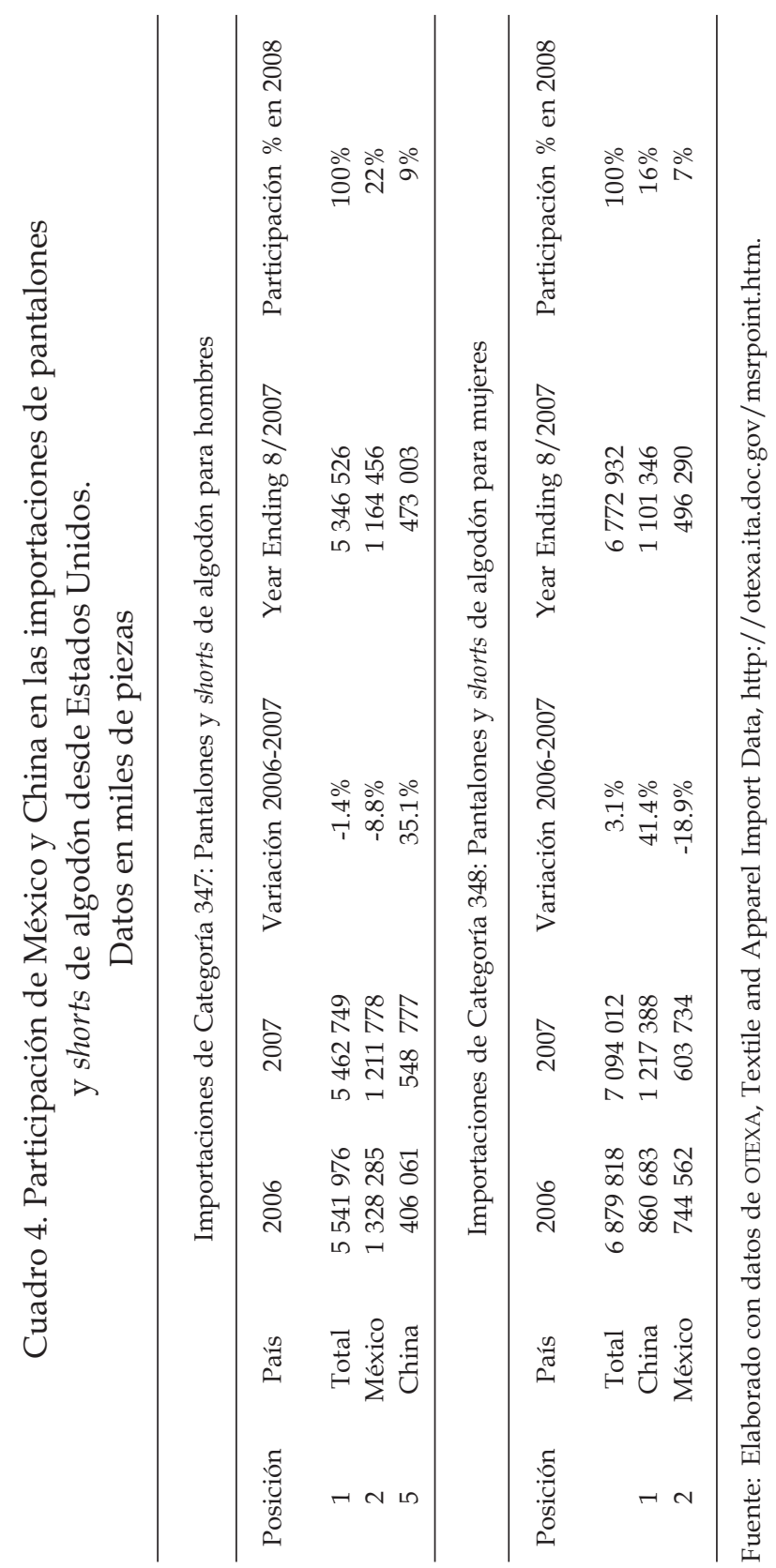


global de las firmas líderes de esta industria, lo cual tiene que ver con las propias pautas de su reestructuración. Asimismo, México no ha logrado desarrollar los nuevos factores de competitividad -flexibilidad, calidad, velocidad y capacidades de diseño y desarrollo del producto- en la forma en que otros países lo han hecho, especialmente China.

\section{Bibliografía}

Appelbaum, Richard P. y Gary Gereffi (1994), "Power and Profits in the Apparel Commodity Chain", en Edna Bonacich et al. (ed.), Global Production: The Apparel Industry in the Pacific Rim, Filadelfia, PA, Temple University Press.

Bonacich Edna y David V. Waller (1994), "Mapping a Global Industry: Apparel Production in the Pacific Rim Triangle", en Edna Bonacich et al. (ed.), Global Production: The Apparel Industry in the Pacific Rim, Filadelfia, PA, Temple University Press.

Colwell, Dara (2002), "Levi's: Made in China?", Alter Net, http:// www.organicconsumers.org/clothes/chineseslevis.cfm.

Downey, Lynn (2007), “A short history of denim", http:// www.levistrauss.com/Downloads/History-Denim.pdf.

Ernst, Dieter (1997), "From Partial to Systemic Globalization: International Production Networks in the Electronic Industry", BRIE Working Paper 98, Berkeley, CA, Berkeley Roundtable on the International Economy (BRIE), University of California, http:/ /brie.berkeley.edu.

García de León, Guadalupe (2008), La inserción de México en la arquitectura cambiante de redes de suministro del vestido hacia Estados Unidos (1985-2003), Colección Jesús Silva Herzog, México, Porrúa.

Gereffi, Gary (1997), "The Reorganization of Production on a World Scale: States, Markets and Networks in the Apparel and Electronics Commodity Chains", en Duncan Campbell (ed.) (1997), Regionalization and Labor Market Interdependence in East and Southeast Asia, Génova, International Institute for Labour Studies. 
Gereffi, Gary (1999), A Commodity Chains Framework for Analyzing Global Industries, Institute of Development Studies, Duke University.

Gibbon, P. (2000), Global Commodity Chains and Economic Upgrading in Less Developed Countries, Centre for Development Research, Copenague, CDR Working Paper 00.2.

Henderson, J. (2002), Globalization on the Ground: Global Production Networks, Competition, Regulation and Economic Development, Center on Regulation and Competition, Institute for Development Policy and Management, University of Manchester, Working Papers Series, Paper No. 38.

Humphrey, John y H. Schmitz (2000), Governance and Upgrading: Linking Industrial Cluster and Global Value Chain Research, IDS Working Paper 120, UK, Institute of Development Studies, University of Sussex.

Kaplinsky, Raphael (2000), Spreading the Gains from Globalization: What can be Learned from Value Chain Analysis?, IDS Working Paper 110, UK, Institute of Development Studies, University of Sussex.

Levi Strauss \& Co. (s/f.), "Form 10-K Annual Reports", Washington, U.S. Securities and Exchange Commission (SEC), Electronical Data Gathering Analysis and Retrieval (EDGAR) database, http:// www.sec.gov/cgi-bin/srch-edgar.

Levi Strauss and Co. (2008), "Supplier List, april 2008", http:// www.levistrauss. com/Downloads/FactoryList.pdf (3 de octubre de 2008).

Newbery, Malcolm (2004), Global Market Review for the Denim and Jeanswear Industries -with Forecasts to 2010, Bromsgrove, Inglaterra, Aroq Limited.

Porter, M. (2000), Ventaja Competitiva. Creación y sostenimiento de un desempeño superior, México, Continental.

Sturgeon, Timothy (2001), How do we Define Chains and Production Networks, US Industrial Performance Center, Massachusetts Institute of Technology.

us Department of Commerce, Office of Textiles and Apparel (OTEXA) (2008), “US Imports, Production, Markets, Import Production Ratios and Domestic Market Shares for Textile and Apparel Product Categories", reporte cuatrimestral. 
ESTUDIOS FRONTERIZOS, VOL. 10, NÚM. 20, JULIO-DICIEMBRE 2009

Us Department of Labor (2000), "Apparel and Other Textile Products", Career Guide to Industries, edición 2000-2001.

US International Trade Commission (USITC) (1999), Industry Trade Summary. Apparel, USITC Publication 3169, Washington, DC, marzo.

us Census Bureau, Economice Census 2002, http:/ / www.census.gov/ econ/census02/. 